\title{
Proteomic Analysis Reveals a New Benefit of Periodic Mechanical Stress on Chondrocytes
}

\author{
Zeng Li Zhen Wang Shun Xu Wenwei Liang Weimin Fan \\ Department of Orthopedics, the First Affiliated Hospital of Nanjing Medical University, Nanjing, China
}

\section{Key Words}

Proteomics analysis • Extracellular matrix • Periodic mechanical stress • Type I collagen • Wnt/ beta-catenin pathway

\begin{abstract}
Background/Aims: In recent years, a variety of studies have been performed to investigate the cellular responses of periodic mechanical stress. In our previous studies, we found that periodic mechanical stress can promote proliferation and matrix synthesis through the integrin beta 1-mediated ERK1/2 pathway, and we used proteomic analysis to detect quantitative changes in chondrocytes under periodic mechanical stress. Despite these results, the effects and mechanisms of periodic mechanical stress are still not fully understood, so in this study we extended our study using phosphoproteomic techniques. Methods: We used phosphoproteomic techniques to detect phosphorylation changes in chondrocytes under periodic mechanical stress and combined the results with the quantitative proteomic data to further explore the underlying mechanisms. Data were obtained by phosphorylation inhibition, quantitative real-time PCR ( $\mathrm{PPCR}$ ) analysis, western blot analysis and immunofluorescence assay. Results: From phosphoproteomic analysis, a total of 1073 phosphorylated proteins and 2054 phosphopeptides were identified. The number of significant differentially expressed proteins and phosphopeptides was 97 and 108, respectively (ratio $>1.20$ or $<0.83$ at $\mathrm{p}$ <0.05). Periodic mechanical stress increased glycogen synthase kinase 3-beta (GSK3-beta) phosphorylation at Y216, promoted the phosphorylation of beta-catenin, decreased betacatenin levels and suppressed the expression of type I collagen. In contrast, inhibition of GSK3-beta by TWS119, which specifically inhibits the phosphorylation of Y216, suppressed the phosphorylation of beta-catenin, which resulted in the accumulation of beta-catenin and an increase in the expression of type I collagen. Conclusions: We successfully constructed differentially expressed phosphoproteomic profiles of rat chondrocytes under periodic mechanical stress, and discovered a potential new therapeutic benefit in which periodic mechanical stress suppressed the formation of type I collagen in the matrix of chondrocytes via phosphorylation of GSK3-beta and beta-catenin.

Z. Li, Z. Wang and S. Xu are co-first authors.

Weimin Fan

and Wenwei Liang

Department of Orthopedics, The First Affliated Hospital of Nanjing Medical University 300 Guang Zhou Road, Nanjing, China

Tel.+86-25-83718836-6780, E-Mail fanweimin@vip.sina.com and liangwenwei123@sina.com
\end{abstract}




\section{Introduction}

Articular cartilage is always exposed to various mechanical stimuli such as dynamic compression, hydrostatic pressure and fluid shear. However, given the normal activity of the joint, dynamic compression is without doubt the most important of these stimuli. Matrix homeostasis of chondrocytes is strongly influenced by dynamic compression, which affects its composition, structure and biomechanical properties [1]. Studies in vivo have shown that matrix biosynthesis in cartilage increased with moderate exercise and decreased during immobilization $[2,3]$.

Proper dynamic compression is not only good for cartilage in vivo, but also provides substantial benefits to chondrocytes in vitro. Periodic mechanical stress in vitro, which mimics dynamic compression in vivo, has been shown to significantly enhance the function of chondrocytes. Some studies have revealed that periodic mechanical stress can promote chondrocyte proliferation, migration and matrix synthesis of type II collagen and aggrecan [4-7]. In our previous studies, we found that a periodic stress field with a pressure range of $0-200 \mathrm{kPa}$ at $0.1 \mathrm{~Hz}$ had the greatest effect on chondrocytes and promoted their proliferation and matrix synthesis through the integrin beta 1 -mediated ERK1/2 pathway $[8,9]$. There are likely other effects of periodic mechanical stress that have not been fully identifid in chondrocytes.

Proteins are the foundation of matrix and perform various functions. Proteomic techniques are continuously being developed to further understand biologic behaviors and are important tools to study the distribution and interaction of all the proteins in cells or organisms $[10,11]$. In order to explore undiscovered effects of periodic mechanical stress on chondrocytes in our previous study, we screened differentially expressed proteins by quantitative proteomic analysis [12]. A total of 5468 proteins were identified and 485 were differential expressed after periodic mechanical stress (168 proteins were upregulated while 317 were downregulated). However, many biologic behaviors rely not only on quantitative changes in protein levels but also on posttranslational modifications (PTMs) such as phosphorylation, acetylation, and glycosylation [13]. Among these modifications, phosphorylation is the most widespread reversible PTM and plays an important role in regulating almost every cellular activity [14]. Thus, in the present study we screened differentially phosphorylated proteins by phosphoproteomic techniques to further explore the effects of periodic mechanical stress on chondrocytes.

\section{Materials and Methods}

\section{Materials}

Sprague-Dawley rats (1 week old) were provided by the Animal Center of Nanjing Medical University. Dulbecco's Modified Eagle Medium (DMEM-low glucose), fetal bovine serum (FBS), phosphate buffered saline (PBS) and trypsin were purchased from Gibco (Grand Island, NY). Collagenase II, type II collagen and C18 Cartridge were obtained from Sigma-Aldrich (St Louis, MO). BCA Protein Assay Kit, RIPA lysis buffer was provided by Biyuntian (Jiangsu, China). SDS, Urea, IAA and DTT were purchased from Bio-rad (Hercules, CA). iTRAQ Reagent-4plex Multiplex Kit was obtained from AB SCIEX (Framingham, MA). $\mathrm{TiO}_{2}$ was obtained from Shimadzu (Kyoto, Japan). Cell Counting Kit-8 (CCK-8) was acquired from Dojindo Laboratories (Kumamoto, Japan). Primers for GAPDH (RQP049537), Col1A1 (RQP054226) and Col2A1 (RQP049248) were provided by GeneCopoeia (Rockville, MD). RNA Extraction Kit, PrimeScript ${ }^{\mathrm{TM}}$ RT Master Mix and SYBR Premix Ex $\mathrm{Taq}^{\mathrm{TM}}$ were acquired from TaKaRa (Shiga, Japan). Anti-collagen I antibody, anti-collagen II antibody, Alexa Fluor $\AA$ 488-conjugated anti-mouse immunoglobulin IgG and Alexa Fluor® 488-conjugated anti-rabbit IgG were supplied by Abcam (Cambridge, UK). Anti-phospho-ERK1/2 antibody, anti-ERK1/2 antibody, antiphospho-GSK3-beta (Y216) antibody, anti-phospho-GSK3-beta (S9) antibody, anti-GSK3-beta antibody, anti-phospho-beta catenin antibody, anti-beta catenin antibody, HRP-goat anti-rabbit IgG and HRP-goat anti-mouse IgG were supplied by Cell Signaling Technology (Beverly, MA). HRP-conjugated GAPDH antibody was provided by Kang Chen (Shanghai, China). TWS119 was obtained from Selleck (Houston, TX). Enhanced chemiluminescence (ECL) kit was purchased from Thermo Fisher Scientific (Waltham, MA).

\section{KARGER}




\section{Cellular Physiology Cell Physiol Biochem 2017;44:1578-1590 \begin{tabular}{ll|l} 
and Biochemistry Published online: December 04, 2017 & $\begin{array}{l}\text { (c) } 2017 \text { The Author(s). Published by S. Karger AG, Basel } \\
\text { www.karger.com/cpb }\end{array}$
\end{tabular}}

Li et al.: New Benefit of Stress on Chondrocytes

Cell culture

Articular hyaline cartilage was isolated from the knees of one-week-old rats under sterile conditions and sliced into pieces $\left(1 \mathrm{~mm}^{3}\right)$ for subsequent digestion. The cartilage was first incubated with $0.25 \%$ trypsin for $30 \mathrm{~min}$, followed by $0.2 \%$ collagenase II for $4 \mathrm{~h}$ at $37^{\circ} \mathrm{C}$. The digested samples were purified with a cell strainer (about $10^{7}$ chondrocytes per rat) and cultured in DMEM-low glucose with 10\% FBS in an incubator (Heraeus BB 5060, Thermo Fisher Scientific) at $37^{\circ} \mathrm{C}$ with $5 \% \mathrm{CO}_{2}$. Chondrocytes were passaged by trypsinization when cells attained over $90 \%$ confluence. When passaged to the second generation, chondrocytes were seeded on slides or plates for the following experiments.

\section{Construction of periodic mechanical stress field}

The periodic stress field with adjustable stress intensity and frequency was constructed by connecting a reciprocating intensifier pump to an air-tight cell culture device (Taixing Experimental Instrument Factory, Jiangsu, China) through a barrier-type pressure transducer (Tianjin Plastics Research Institute, Tianjin, China) as described previously [8, 9]. After passage to the second generation, chondrocytes were seeded at a density of $10^{5}$ cells $/ \mathrm{ml}$ on glass slides (diameter: $24 \mathrm{~mm}$ ) or culture bottles (area: $75 \mathrm{~cm}^{2}$ ). Experiments were performed when cells were approximately $70 \%$ confluent. As in our previous experiments, a pressure range of 0-200 $\mathrm{kPa}$ applied at $0.1 \mathrm{~Hz}$ frequency was used in this study $[8,9]$.

\section{SDS-PAGE separation and sample preparation}

Total protein from cells was obtained by extraction in SDT lysis buffer (4\%SDS, $1 \mathrm{mM}$ DTT, $150 \mathrm{mM}$ Tris- $\mathrm{HCl}, \mathrm{pH}$ 7.6). The lysate was sonicated and centrifuged at 14, $000 \mathrm{~g}$ for $40 \mathrm{~min}$ and the supernatant was quantified with the BCA Protein Assay Kit. Then, $20 \mu \mathrm{g}$ of protein from each sample (three individual samples per group) were mixed with $5 X$ loading buffer (Biyuntian, Jiangsu, China) and boiled for 5 min. The proteins were separated by $12.5 \%$ SDS-PAGE (constant current $15 \mathrm{~mA}, 60 \mathrm{~min}$ ) and visualized by Coomassie Blue staining. Pooled replicates (three individual samples per group from nine rats) were used in the present study $[15,16]$. Protein digestion was performed according to the FASP procedure [17]. First, $200 \mu \mathrm{g}$ of protein from each sample were incorporated into $100 \mathrm{mM}$ DTT. The detergent, DTT and other components were washed in UA buffer ( $8 \mathrm{M}$ Urea, $150 \mathrm{mM}$ Tris-HCl, pH 8.0) by repeated ultrafiltration. Then, $100 \mu \mathrm{l}$ IAA (100 mM IAA in UA buffer) were added and the filters were incubated for 30 min in darkness. The filters were washed three times with UA buffer and then twice in DS buffer (50 mM triethylammonium bicarbonate buffer, pH 8.5). Finally, the filter-bound proteins were digested with $2 \mu$ g trypsin in $40 \mu$ DS buffer overnight at $37^{\circ} \mathrm{C}$ and the resulting peptides were collected as a filtrate. The peptide content was estimated by UV light spectral density at $280 \mathrm{~nm}$.

\section{ITRAQ Labeling and enrichment of phosphopeptides by $\mathrm{TiO}_{2}$ beads}

For labeling, the peptide mixture was labeled using iTRAQ reagent dissolved in $70 \mu$ ethanol according to the manufacturer's instructions. Samples were labeled with the iTRAQ tags as follows: control group (113, 114 and 115) and pressure group (116, 117 and 118). The labeled peptides were multiplexed and vacuum dried [18]. The labeled peptides were concentrated and resuspended in $500 \mu \mathrm{l}$ DHB buffer ( $\mathrm{Sigma}_{\text {). }} \mathrm{TiO}_{2}$ beads were then added and agitated twice for $40 \mathrm{~min}$. The beads were washed with washing buffer I ( $30 \%$ ACN, 3\% TFA) three times and washing buffer II (80\% ACN, 0.3\% TFA) three times. The phosphopeptides were eluted with $50 \mu \mathrm{l}$ of elution buffer ( $\left.40 \% \mathrm{ACN}, 15 \% \mathrm{NH}_{4} \mathrm{OH}\right)$ followed by lyophilization and MS analysis [19].

\section{Mass spectrometry}

The mixture of phosphopeptides was dissolved in 0.1\% TFA and then injected for nanoLC-MS/MS analysis using Q Exactive MS with Easy nLC (Thermo Fisher Scientific). The peptide mixture was loaded onto a C18-reverse phase column (15 cm long, $75 \mu \mathrm{m}$ inner diameter, RP-C18 $3 \mu \mathrm{m}$, packed in-house) in buffer A ( $0.1 \%$ formic acid) and separated with a linear gradient of buffer B ( $80 \%$ ACN, $0.1 \%$ formic acid) at a flow rate of $250 \mathrm{nl} / \mathrm{min}$ controlled by IntelliFlow technology over $240 \mathrm{~min}$. The peptides were eluted with a gradient of $0-55 \%$ buffer B for $220 \mathrm{~min}, 55 \%-100 \%$ buffer B for $8 \mathrm{~min}$ and held in 100\% buffer B for 12 min [20]. For MS analysis, peptides were analyzed in positive ion mode. MS data was acquired using a data-dependent top10 method dynamically choosing the most abundant precursor ions from the survey scan (300-1800 m/z) for HCD fragmentation. Automatic gain control target was set to 3e6, and 
Li et al.: New Benefit of Stress on Chondrocytes

maximum injection time to $10 \mathrm{~ms}$. Dynamic exclusion duration was $40.0 \mathrm{~s}$. Survey scans were acquired at a resolution of 70, 000 at m/z 200, while resolution for HCD spectra was set to 17, 500 at m/z 200 and isolation width was $2 \mathrm{~m} / \mathrm{z}$. Normalized collision energy was $30 \mathrm{eV}$ and the underfill ratio, which specifies the minimum percentage of the target value likely to be reached at maximum fill time, was defined as $0.1 \%$. The instrument was run with peptide recognition mode enabled [21].

\section{Data analysis of mass spectrometry}

MS/MS spectra were searched using the MASCOT engine (version 2.2; Matrix Science, London, UK) embedded into Proteome Discoverer 1.4 (Thermo Scientific, San Jose, CA) against the Uniprot_rat database (uniprot_rat_34170_20150508.fasta) and the decoy database. For protein identification, the following options were used. Peptide mass tolerance: $\pm 20 \mathrm{ppm}$; MS/MS tolerance: $0.1 \mathrm{Da}$; enzyme: trypsin; missed cleavage: 2; fixed modification: carbamidomethyl (C), iTRAQ8plex (N-term) and iTRAQ8plex (K); variable modification: oxidation (M) and phosphorylation (S/T/Y). The score threshold for peptide identification was set at a $1 \%$ false discovery rate (FDR), and values were rejected if not all quan channels were present. All peptide ratios were normalized to 1 by the median protein ratio. The following criteria were required to consider a protein for further phospho RS analysis: phospho RS score $>50$ and phospho RS site probabilities $>75 \%$ (Protein Discoverer 1.4). Changes with either a ratio $>1.20$ or $<0.83$ and $p<0.05$ were considered significant in proteomic analysis [22-25].

\section{Bioinformatics analysis}

Gene ontology (GO) enrichment analysis was performed against the Swiss-Prot mammal database (rat) using NCBI BLAST+ software (ncbi-blast-2.2.28+-win32.ext) to find homologous sequences and allow the identified sequences to be functionally annotated. The protein interaction analysis was performed using online STRING (https://string-db.org/, version 10.0).

\section{Reverse transcription-quantitative polymerase chain reaction (RT-qPCR) analysis}

Total RNA was extracted using an RNA extraction kit and the concentration and purity was measured using a spectrophotometer (Nanodrop 2000; Thermo Fisher Scientific). RNA with a 260/280 ratio of 1.8-2.0 was reverse transcribed into cDNA using PrimeScript RT Master Mix. qPCR analysis was performed in a 10$\mu \mathrm{l}$ mixture using the LightCycler 480 System (Roche Diagnostics, Basel, Switzerland). Briefly, $1 \mu \mathrm{l}$ cDNA was mixed with $1 \mu \mathrm{l}$ specific primers, $4 \mu \mathrm{l}$ water and $5 \mu$ l SYBR Premix Ex Taq and amplified under the following cycling conditions: $95^{\circ} \mathrm{C}$ for $30 \mathrm{sec}$, followed by 40 cycles at $95^{\circ} \mathrm{C}$ for $5 \mathrm{sec}$ and $60^{\circ} \mathrm{C}$ for $20 \mathrm{sec}$, and a final extension at $72^{\circ} \mathrm{C}$ for $10 \mathrm{~min}$. Gene expression of COL1A1 and COL2A1 was normalized to GAPDH expression using the $2^{-\triangle \Delta \mathrm{Ct}}$ method, as previously described [26]. Each experiment was repeated at least three times.

\section{Western blot analysis}

Total protein was obtained by extraction of cells with RIPA lysis buffer (Beyotime Institute of Biotechnology, Haimen, China) containing 1\% PMSF and the BCA assay was used to determine protein concentrations. Protein samples $(30 \mu \mathrm{g})$ were separated by $10 \%$ SDS-PAGE and transferred to polyvinylidenefluoride membranes. After blocking with 5\% skim milk in TBST for $1 \mathrm{~h}$, membranes were incubated with primary antibodies at $4^{\circ} \mathrm{C}$ overnight and with secondary antibodies at ambient temperature for $1 \mathrm{~h}$. Blots were visualized with an enhanced ECL kit using a gel imaging system (UVP LLC, Upland, CA) and measured by Image Lab software (Sausage Software, Melbourne, Australia). Each experiment was repeated at least three times.

\section{Immunofluorescence assay}

Cells seeded on slides (diameter: $24 \mathrm{~mm}$ ) were first fixed with $4 \%$ paraformaldehyde for $30 \mathrm{~min}$ at room temperature. After washing with PBS and permeabilization of the cytomembrane, the cells were incubated with primary antibodies at $4{ }^{\circ} \mathrm{C}$ overnight and with secondary antibodies at ambient temperature for $50 \mathrm{~min}$. The cells were counterstained with DAPI for $10 \mathrm{~min}$ in the dark. Images were taken by fluorescent microscope (Nikon, Tokyo, Japan).

\section{Statistical analysis}

Statistical analyses were performed using IBM (Armonk, NY) SPSS 14.0 software and results are presented as means \pm standard error (SE). Student's t-test and analysis of variance with Student-Newman- 
Keuls post hoc tests were used to determine statistical significance. P-values $<0.05$ were considered to indicate a statistically significant difference.

\section{Results}

Phosphoproteomic analysis of periodic mechanical stress on chondrocytes

Chondrocytes from the second generation were divided into two groups. The control group was cultured under static conditions, while the pressure group was subjected to stress varying from 0 to $200 \mathrm{kPa}$ at $0.1 \mathrm{~Hz}$ for $8 \mathrm{~h}$ per day for 3 days. From the phosphoproteomic data, a total of 1073 phosphorylated proteins and 2054 phosphopeptides were identified. Analysis of the frequency distribution histogram of quantitative data (using P1 as an example) showed that the fold changes ( $\log _{2}$ ) of phosphopeptides were distributed normally (Fig. 1A). The number of significant differentially expressed proteins was 97 and the number of phosphopeptides was 108 (ratio $>1.20$ or $<0.83$ and $\mathrm{p}<0.05$ ). The bioinformatics analysis indicated that these 97 differentially expressed proteins were involved in 1404 GO function entries (Blast2GO, Version 2.7.2; Fig. 1B). GO analysis for identified proteins revealed a strong enrichment for GO terms associated with binding, biological regulation, cellular component organization, cellular process, developmental process, metabolic process, multicellular organismal process, pigmentation and response to stimulus.

Fig. 1. Phosphoproteomic analysis of periodic mechanical stress on chondrocytes. (A) Frequency distribution histogram of quantitative data using P1 as an example. The fold changes $\left(\log _{2}\right)$ of phosphopeptides were distributed normally. (B) Gene Ontology (GO) classification of differentially accumulated proteins (ratios $>1.20$ or $<0.83$ at $\mathrm{p}<0.05$ ).

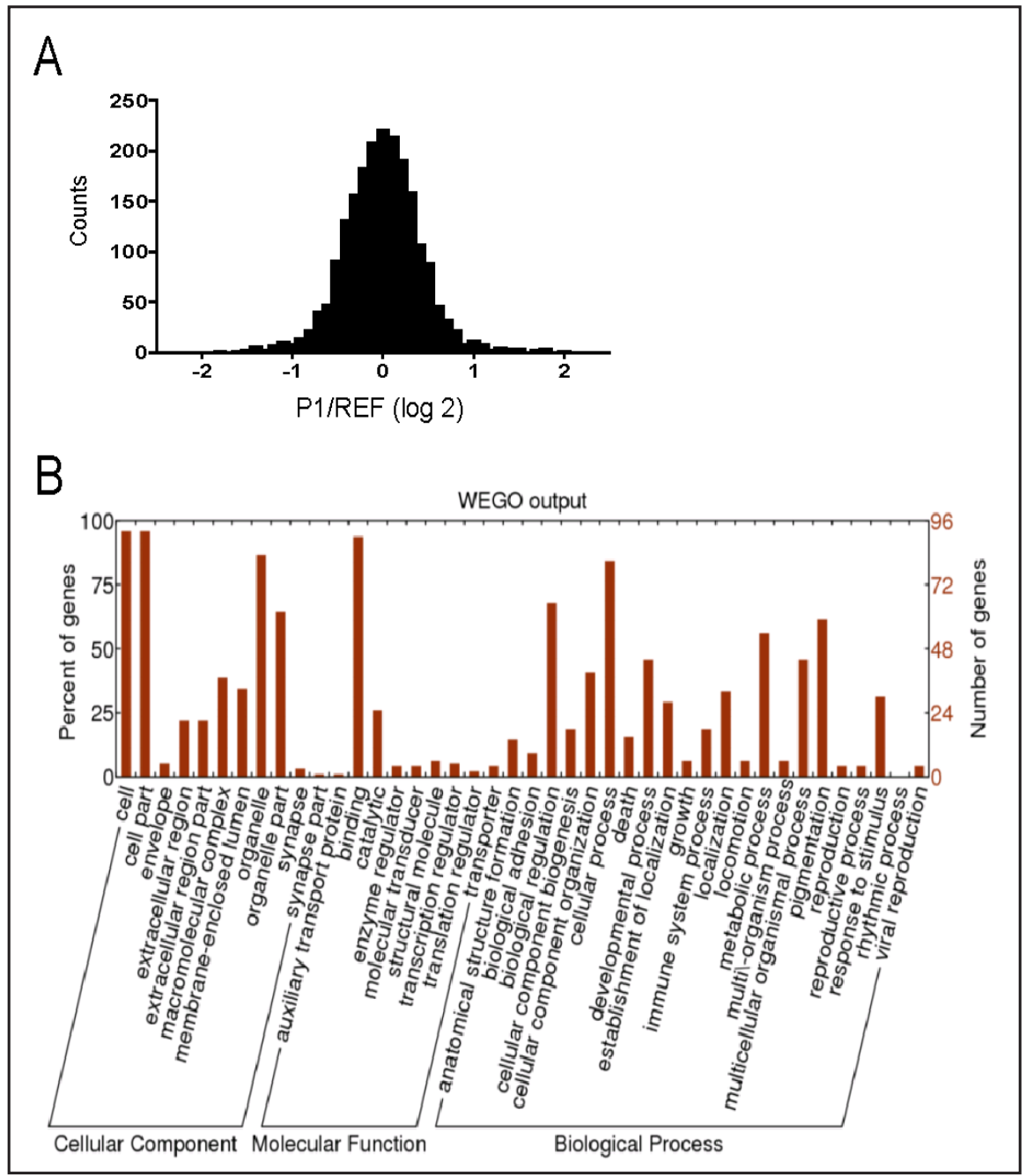


Fig. 2. Periodic mechanical stress promoted ERK1/2 phosphorylation. (A) Fold change ratios of p-ERK1 and p-ERK2. Phosphorylated levels of ERK1 and ERK2 increased significantly after periodic mechanical stress in chondrocytes (the fold change ratios were 1.521 and 1.278 , respectively). ${ }^{*}$ Fold change $>1.2$ at $\mathrm{p}<0.05$ compared to control $(\mathrm{n}=3$, bar=SE). (B) Representative western blots and quantification of p-ERK1/2 and ERK1/2. In western blot analysis, phosphorylation of ERK1 and ERK2 was significantly increased after periodic mechanical stress in agreement with the results of phosphoproteomic analysis. N, control group; P, pressure group. ${ }^{*} \mathrm{p}<0.05$ compared to control $(n=3$, bar=SE).

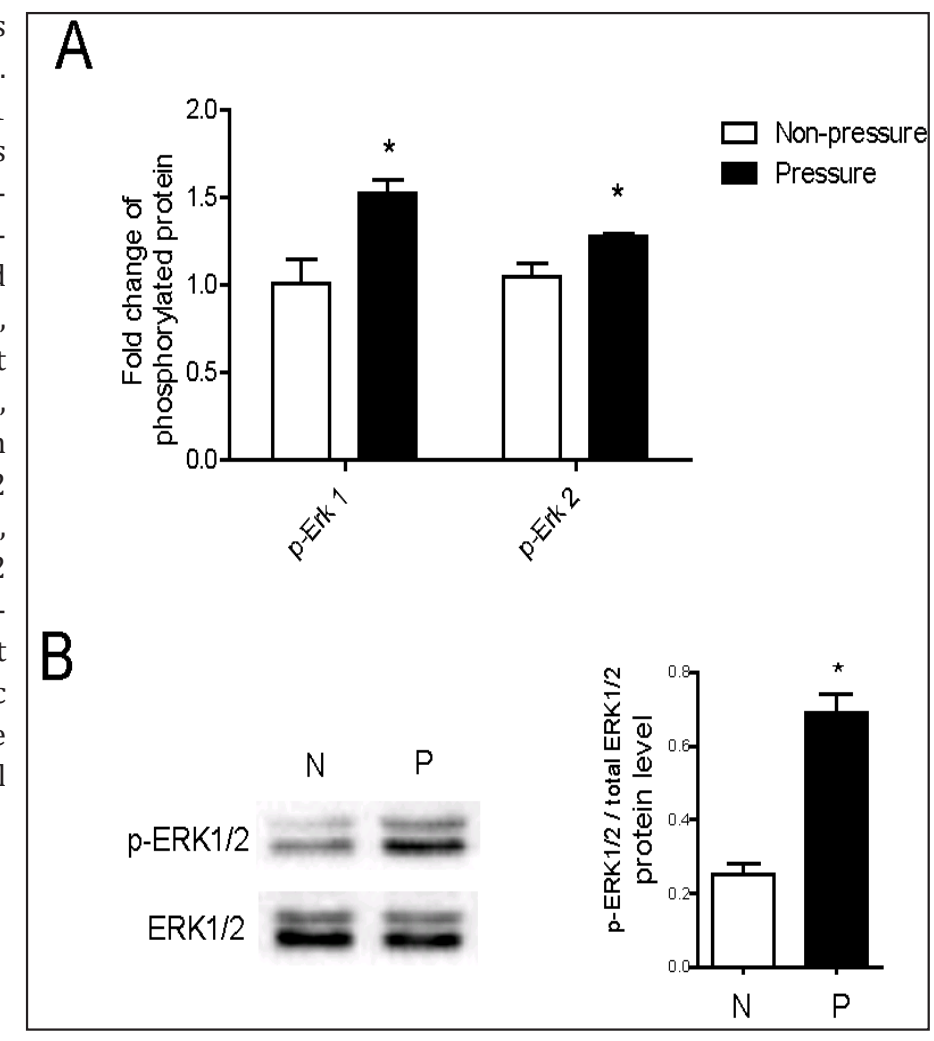

Table 1. Fold changes in collagen related to fibrosis according to quantitative protein analysis. (A, control group; B, pressure group)

\begin{tabular}{llll}
\hline Accession Number & Description & B/A & p-value \\
\hline PO2454 & Col1a1 & 0.523 & 0.001 \\
F1LS40 & Col1a2 & 0.482 & 0.000 \\
P13941 & Col3a1 & 0.680 & 0.003 \\
Q9JI03 & Col5a1 & 0.704 & 0.001 \\
D3ZUL3 & Col6a1 & 0.768 & 0.006 \\
P20909 & Col11a1 & 0.692 & 0.002 \\
D3ZZT9 & Col14a1 & 0.534 & 0.004 \\
F1LR02 & Col18a1 & 0.657 & 0.035 \\
\hline
\end{tabular}

Periodic mechanical stress promoted ERK1/2 phosphorylation in chondrocytes

In our previous studies, we found that periodic mechanical stress increased chondrocyte proliferation through the integrin beta 1-ERK1/2 signaling cascade which triggered the phosphorylation of ERK1/2 $[8,9]$. In the present study, phosphoproteomic analysis also indicated that ERK1 and ERK2 were phosphorylated with significant fold change ratios of 1.521 and $1.278(\mathrm{p}<0.05$; Fig. $2 \mathrm{~A})$. We then verified the results by western blot analysis, which showed that the levels of phosphorylated ERK1 and ERK2 were significantly increased after periodic mechanical stress (Fig. 2B). This was consistent with our previous results and demonstrated the reliability of the phosphoproteomic analysis.

Periodic mechanical stress suppressed type I collagen in chondrocytes

From the previous quantitative proteomic data [12], we found for the first time a phenomenon in which protein levels of type I collagen and some other types of collagen related to fibrosis, including type III, V and VI collagen, were significantly suppressed under periodic mechanical stress (the fold change ratios were $0.680,0.704$ and 0.768 , respectively; $\mathrm{p}<0.05$ ), while the protein level of type II collagen showed no significant change (Table 1; Fig. $4 \mathrm{~A})$. In addition, the protein interaction analysis indicated that there were close interactions 
Fig. 3. Protein interaction analysis of type I and related collagens (https:// string-db.org/, version 10.5). Protein interaction analysis of type I and related collagens which were significantly changed after periodic mechanical stress (ratios $>1.20$ or $<0.83$ at $\mathrm{p}<0.05$ ). The combined association scores varied from 0.428 to 0.998 . The mean score was 0.832 .

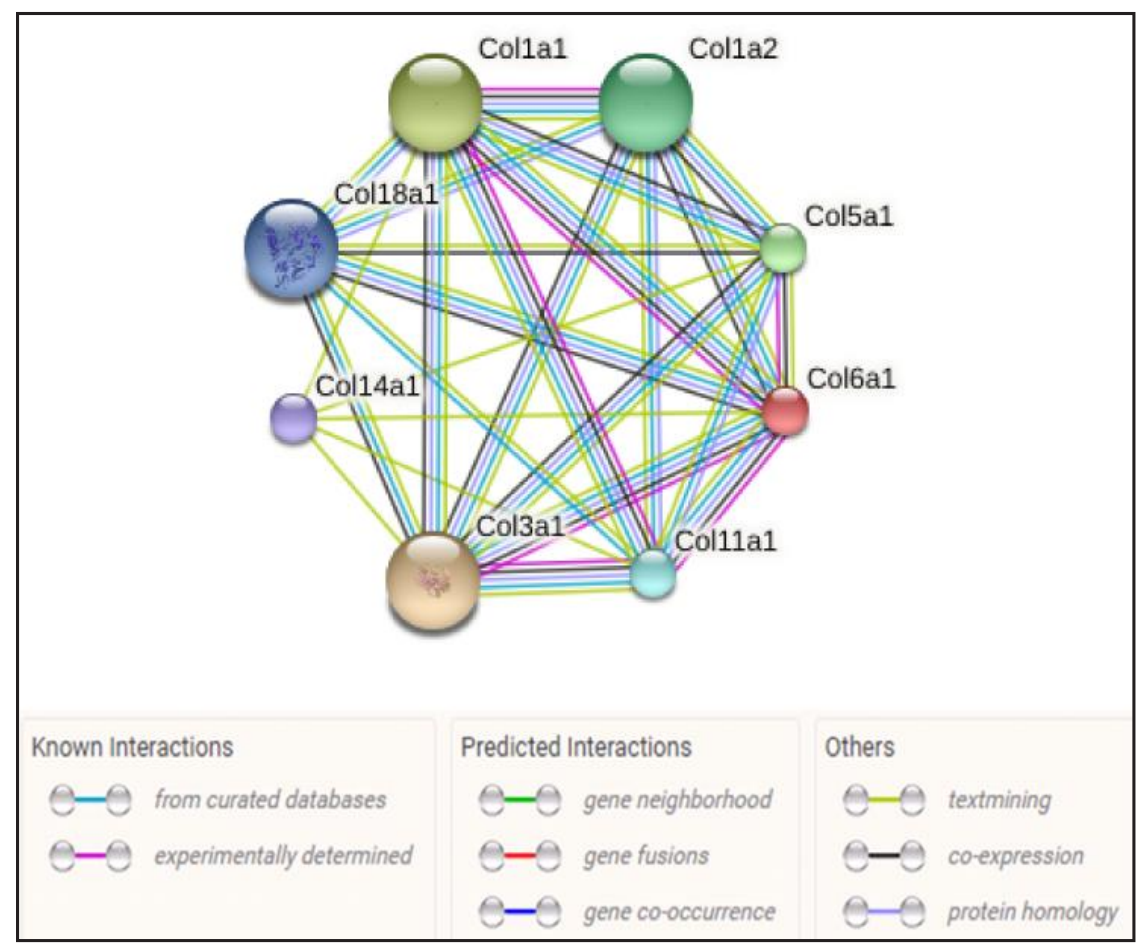

Fig. 4. Periodic mechanical stress suppressed type I collagen. (A) Fold change ratios of type I collagen and type II collagen by quantitative proteomic analysis. Protein levels of type Ia1 and type Ia2 collagen decreased significantly after periodic mechanical stress (fold change ratios were 0.523 and 0.482 , respectively; $p<0.05$ ) while type II collagen remained constant (fold change ratio was 0.962). * Fold change $<0.83$ at $\mathrm{p}<0.05$ compared to control $(n=3$, bar=SE). (B, C) Representative western blots and quantification of type I and type II collagen. The protein levels of type I collagen decreased signifi-

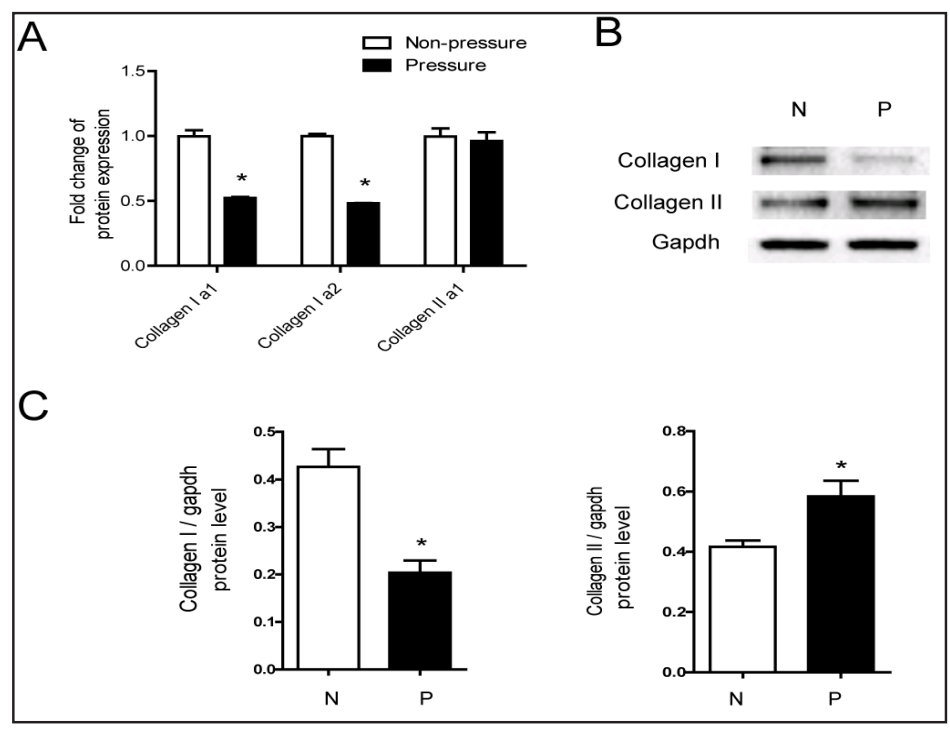
cantly after periodic mechanical stress, while type II collagen protein levels increased. The change in protein levels was consistent with the results of proteomic analysis. N, control group; P, pressure group. ${ }^{*} \mathrm{p}<0.05$ compared to control ( $n=3$, bar=SE).

among these collagens that might change accordingly with each other. The combined association scores varied from 0.428 to 0.998 and the mean score was 0.832 (Fig. 3). We then tested the results by western blot analysis, which indicated that protein levels of type I collagen decreased significantly after periodic mechanical stress, while type II collagen protein levels increased, basically in agreement with the results of our proteomic analysis (Fig. 4B and C). This indicated that periodic mechanical stress suppressed type I collagen in chondrocytes, although we still did not know the underlying mechanism of this phenomenon. Therefore, we scanned our phosphoproteomic analysis data for clues. 
Fig. 5. Periodic mechanical stress stimulated GSK3-beta and betacatenin phosphorylation. (A) Fold change ratios of GSK3-beta and betacatenin by quantitative protein analysis. The protein level of GSK3-beta remained constant (fold change ratio was 1.023 ), while the protein level of beta-catenin decreased slightly (fold change ratio was $0.896>0.830$; $\mathrm{p}<0.05$ ). * Fold change $>1.2$ or $<0.83$ at $\mathrm{p}<0.05$ compared to control $(n=3$, bar=SE). (B) Fold change ratios of p-GSK3-beta and p-betacatenin by phosphoproteomic analysis. The phosphorylated levels of GSK3-

beta and beta-catenin increased significantly after periodic mechanical stress in chondrocytes (fold change ratios were 1.366 and 1.409 , respectively). * Fold change $>1.2$ or $<0.83$ at $p<0.05$ compared to control $(n=3$, bar=SE). (C) Representative western blots and quantification of GSK3-beta and beta-catenin. Protein levels of p-GSK3-beta (Y216) and p-beta-catenin increased significantly after periodic mechanical stress, while beta-catenin protein levels decreased. The change in protein levels was consistent with the result of proteomic analysis. N, control group; P, pressure group. * $\mathrm{p}<0.05$ compared to control ( $n=3$, bar=SE).

Periodic mechanical stress stimulated GSK3-beta and beta-catenin phosphorylation in chondrocytes

In conjunction with the decrease in type I collagen, phosphoproteomic analysis in the present study indicated that GSK3-beta (Y216) and beta-catenin were phosphorylated after periodic mechanical stress (the fold change ratios were 1.336 and 1.409, respectively; $\mathrm{p}<0.05$ ) with no significant quantitative change in non-phosphorylated protein levels (Fig. $5 \mathrm{~A}$ and $\mathrm{B})$. We tested this result by western blot analysis and showed that the protein levels of p-GSK3-beta (Y216) and p-beta-catenin increased significantly after periodic mechanical stress, while beta-catenin protein levels decreased, basically in agreement with the results of our proteomic analysis (Fig. 5C). GSK3-beta and beta-catenin are two critical proteins in the Wnt/beta-catenin signaling pathway which is closely related to fibrosis in various tissues and organs $[27,28]$. Thus, we hypothesized that periodic mechanical stress suppressed type I collagen via the phosphorylation of GSK3-beta and beta-catenin.

Periodic mechanical stress suppressed type I collagen via phosphorylation of GSK3-beta and beta-catenin

Chondrocytes were divided into four groups after passage to the second generation. Two groups were treated with DMSO while the other two groups were treated with TWS119 (10 $\mu \mathrm{M}$ in DMSO) [29], an inhibitor of GSK3-beta (especially phosphorylation of Y216) [30], for $24 \mathrm{~h}$ under serum-free conditions. One each of the two control and treated groups were then cultured under static conditions or with periodic mechanical stress. Under periodic mechanical stress, p-GSK3-beta (Y216) and p-beta-catenin levels were enhanced in the control group, while in the treated groups, TWS119 inhibited the stress-induced increase in 
Fig. 6. TWS119 inhibited phosphorylation of GSK3-beta (Y216) and enhanced expression of beta-catenin. Under periodic mechanical stress, protein levels of p-GSK3-beta (Y216) and p-beta-catenin increased, while betacatenin decreased. After inhibition of GSK3-beta by TWS119, the protein levels of p-GSK3-beta, GSK3-beta and p-betacatenin decreased, while beta-catenin increased. * $\mathrm{p}<0.05$ compared to the control group without mechanical stress. ** $\mathrm{p}<0.05$ compared to the control group treated with TWS119 in the absence of mechanical stress $(n=3$, bar=SE).

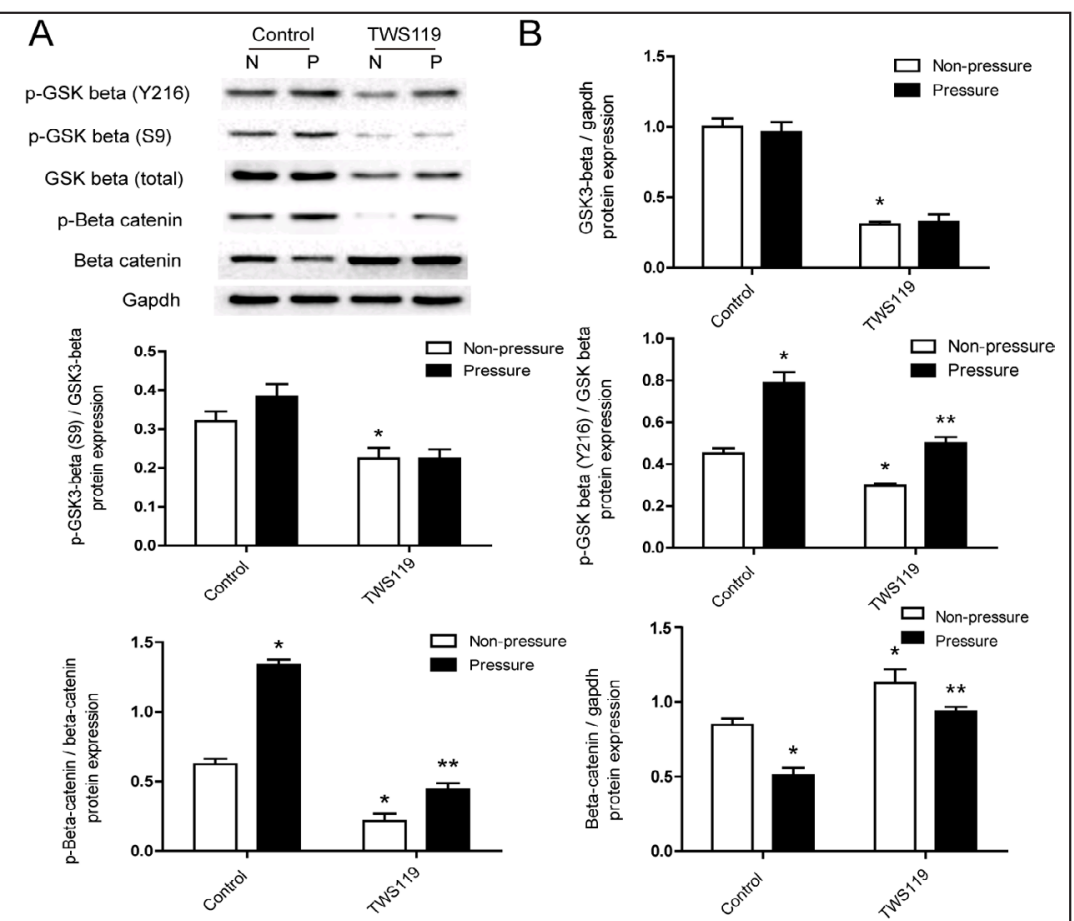

Fig. 7. Periodic mechanical stress suppressed type I collagen in the matrix, while TWS119 inhibited the suppression. (A) mRNA levels of type I and type II collagen. Periodic mechanical stress suppressed the mRNA levels of type I collagen, while TWS119 promoted its expression. (B) Representative western blots and quantification of type I collagen and type II collagen. (C) Representative images of immunostaining for type I and type II collagen. Periodic mechanical stress suppressed type I collagen in the matrix, while TWS119 abrogated the inhibition. However, TWS119 did not influence the synthesis of type II collagen. ${ }^{*}$ p $<0.05$ compared to the control group without mechanical stress. ${ }^{* *} \mathrm{p}<0.05$ compared to the control group treated with TWS119 in the absence of mechanical stress ( $n=3$, bar $=S E)$.
A
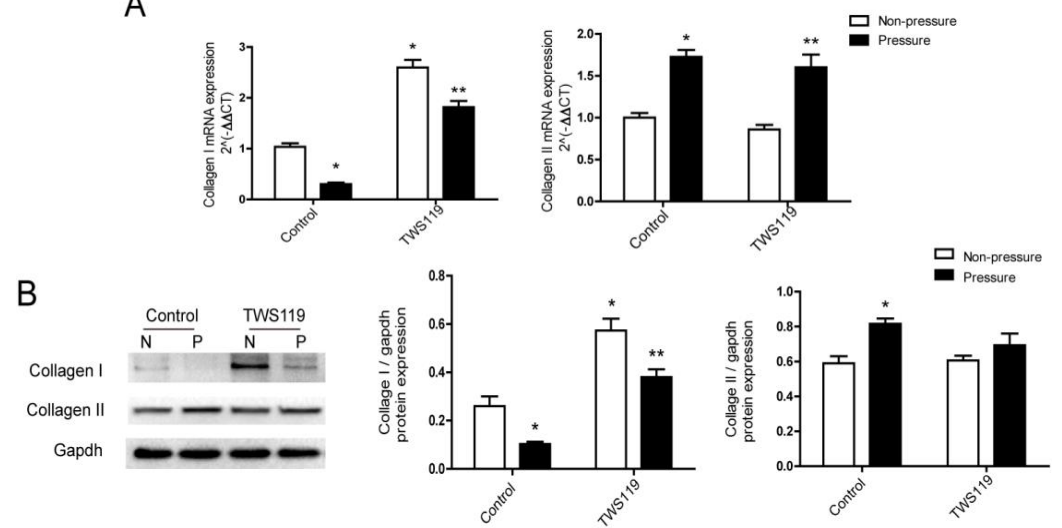

C
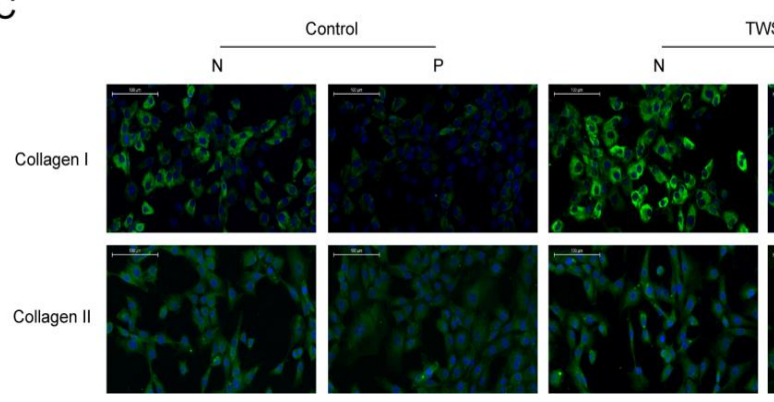

TWS119

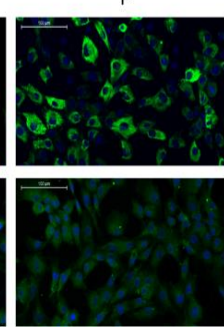


phosphorylation of both proteins. In addition, TWS119 increased the level of beta-catenin both in the absence and during application of periodic stress (Fig. 6). In the analysis of matrix collagens by qPCR, western blot and immunofluorescence assays, periodic mechanical stress suppressed the synthesis of type I collagen, while TWS119 inhibited the phenomenon. These results indicated that periodic mechanical stress suppressed type I collagen via phosphorylation of GSK3-beta and beta-catenin (Fig. 7).

\section{Discussion}

Cells contain a wide variety of proteins and peptides that are the final effectors of diverse biological functions and may be hard to detect. However, proteomic techniques developed in recent years enable the meticulous detection of hundreds of thousands of proteins and peptides in a single experiment and can provide important information regarding protein identification, quantification, and modifications as well as interactions [10].

In the present study, we detected changes in quantity and modifications (phosphorylation) of proteins in chondrocytes under periodic mechanical stress. In our quantitative protein analysis, we found that the protein level of type I collagen, which is always present in fibrocartilage, was significantly suppressed, while the protein level of type II collagen required in articular cartilage showed no significant change after periodic mechanical stress [31]. Not only was type I collagen reduced, but type III, type V, type VI and other related collagens which are closely connected to fibrosis were also decreased after periodic mechanical stress. Among these, type V and type VI collagen serve as reservoirs for fibrogenic-related molecules and thus regulate the availability for fibrogenesis and fibrolysis [32]. However, many of the pathways that are relevant to these mechanisms rely on PTMs such as phosphorylation and not just on quantitative changes in protein levels $[13,14]$, so we had no idea how to explain the changes occurring during periodic mechanical stress until we performed the phosphoproteomic analysis. In the present study, GSK3-beta (Y216) and beta-catenin were phosphorylated to a significantly greater extent after periodic mechanical stress.

Over the past decade, increasing evidence has indicated the critical role of the Wnt/ beta-catenin signaling pathway in the development of organ fibrosis, including skin, lung, liver and renal fibrosis [28, 33]. In animal models, perturbation of the Wnt/beta-catenin signaling pathway, especially GSK3-beta, appears to aggravate or ameliorate fibrosis in a variety of different tissues [34,35]. In this pathway, beta-catenin is a key regulator which can translocate into the nucleus and bind to TCF/LEF to stimulate the transcription of Wnt target genes, including fibrosis-related genes. Beta-catenin can be phosphorylated by a destruction complex which is composed of GSK3-beta and then ultimately destroyed by the proteasome [36]. GSK3-beta is an enzyme which is activated by phosphorylation at Y216 and inhibited by phosphorylation at S9 $[37,38]$. Thus, phosphorylation at Y216 can activate GSK3-beta in the destruction complex and promote the phosphorylation and destruction of beta-catenin to decrease transcription of fibrosis-related genes. In our experiment, we used TWS119 to inhibit phosphorylation of Y216 on GSK3-beta. It was found that the phosphorylation of beta-catenin decreased while levels of beta-catenin increased, as did type I collagen. In summary, periodic mechanical stress increased GSK3-beta (Y216) phosphorylation, promoted the phosphorylation of beta-catenin, decreased beta-catenin levels and suppressed the expression of type I collagen. In contrast, inhibition of GSK3-beta by TWS119, which specifically inhibits the phosphorylation of Y216, suppressed the phosphorylation of betacatenin and resulted in the accumulation of beta-catenin and an increase in the expression of type I collagen. These results indicated that periodic mechanical stress suppressed the formation of type I collagen via phosphorylation of GSK3-beta (Y216) and beta-catenin in the Wnt/beta-catenin signaling pathway.

Articular cartilage is subjected to a wide range of complex mechanical stress during the action of the joints. Mechanical stress plays an important role in the biosynthesis, 
remodeling, degradation and repair of articular cartilage [1]. Our previous research indicated that periodic mechanical stress, which mimics the physiological loading pattern produced by activity, could promote chondrocyte proliferation and matrix synthesis through the integrin beta 1-ERK1/2 signaling pathway $[8,9]$. In the present study, we found that periodic mechanical stress offers the additional benefit of suppressing the formation of type I collagen in the matrix of chondrocytes. This finding may solve the problem of dedifferentiation of chondrocytes, resulting in loss of chondrocyte phenotypes and characteristics: for example, expressing more type I collagen but not type II collagen during monolayer or 3D culture $[39,40]$. Moreover, this finding may not only provide a method to promote the quality of tissue-engineered cartilage [41], but also guide postoperative rehabilitation after surgical interventions for serious cartilage defects. Currently available surgical interventions include microfracture, autologous or allogeneic osteochondral grafts and autologous chondrocyte implantation $[42,43]$. However, almost all interventions have more or less the same problem of fibro-like cartilage: excessive type I collagen production in regenerating cartilage [44-46]. Fibro-like cartilage cannot resist mechanical stress to the same extent as normal hyaline cartilage with type II collagen and is thus vulnerable to injury $[45,47]$. Since periodic mechanical stress can suppress the formation of type I collagen, early load bearing motion after surgery, which provides a type of periodic mechanical stress in the early stage of recovery, may be of benefit for cartilage regeneration.

\section{Conclusion}

we successfully constructed the differentially expressed phosphoproteomic profiles of rat chondrocytes under periodic mechanical stress. We also observed a new benefit in which periodic mechanical stress suppressed the formation of type I collagen in the matrix of chondrocytes via phosphorylation of GSK3-beta (Y216) and beta-catenin. This finding may not only lead to development of novel therapies to promote the quality of tissue-engineered cartilage, but also supports the application of load bearing motion early after surgical interventions for serious cartilage defects.

Although our report is the first to our knowledge to demonstrate that periodic mechanical stress can suppress the formation of type I collagen via phosphorylation of GSK3-beta and beta-catenin, the underlying mechanism requires further exploration. In the future, we look forward to the development of additional applications of periodic mechanical stress in the field of cartilage regeneration.

\section{Acknowledgements}

This work was supported by the National Natural Science Foundation (grant No.: 81672169, 81472079, 81601897).

\section{Disclosure Statement}

The authors declare no competing interests.

\section{References}

\footnotetext{
1 Demarteau 0, Wendt D, Braccini A, Jakob M, Schafer D, Heberer M, Martin I: Dynamic compression of cartilage constructs engineered from expanded human articular chondrocytes. Biochem Biophys Res Commun 2003;310:580-588.
} 


\section{Cellular Physiology Cell Physiol Biochem 2017;44:1578-1590 \begin{tabular}{ll|l} 
and Biochemistry Published online: December 04, 2017 & $\begin{array}{l}\text { C } 2017 \text { The Author(s). Published by S. Karger AG, Basel } \\
\text { www.karger.com/cpb }\end{array}$ \\
\hline
\end{tabular}

2 Vanwanseele B, Lucchinetti E, Stussi E: The effects of immobilization on the characteristics of articular cartilage: Current concepts and future directions. Osteoarthr Cartilage 2002;10:408-419.

-3 Palmoski M, Perricone E, Brandt KD: Development and reversal of a proteoglycan aggregation defect in normal canine knee cartilage after immobilization. Arthritis Rheum 1979;22:508-517.

-4 Pingguan-Murphy B, Nawi I: Upregulation of matrix synthesis in chondrocyte-seeded agarose following sustained bi-axial cyclic loading. Clinics (Sao Paulo) 2012;67:939-944.

5 Wang PY, Chow HH, Lai JY, Liu HL, Tsai WB: Dynamic compression modulates chondrocyte proliferation and matrix biosynthesis in chitosan/gelatin scaffolds. J Biomed Mater Res B Appl Biomater 2009;91:143-152.

6 Liang W, Li Z, Wang Z, Zhou J, Song H, Xu S, Cui W, Wang Q Chen Z, Liu F, Fan W. Periodic Mechanical Stress INDUCES Chondrocyte Proliferation and Matrix Synthesis via CaMKII-Mediated Pyk2 Signaling. Cell Physiol Biochem 2017;42:383-396.

7 He P, Shen N, Gao G, Jiang X, Sun H, Zhou D, Xu N, Nong L, Ren K. Periodic Mechanical Stress Activates PKC $\delta$ Dependent EGFR Mitogenic Signals in Rat Chondrocytes via PI3K-Akt and ERK1/2. Cell Physiol Biochem. 2016;39:1281-1294.

8 Ren K, Liu F, Huang Y, Liang W, Cui W, Wang Q Fan W: Periodic mechanical stress activates integrin $\beta 1$ dependent Src-dependent PLC $\gamma 1$-independent Rac1 mitogenic signal in rat chondrocytes through ERK1/2. Cell Physiol Biochem 2012;30:827-842.

-9 Liang W, Ren K, Liu F, Cui W, Wang Q, Chen Z, Fan W: Periodic mechanical stress stimulates the FAK mitogenic signal in rat chondrocytes through ERK1/2 activity. Cell Physiol Biochem 2013;32:915-930.

10 Butterfield DA, Gu L, Di Domenico F, Robinson RA: Mass spectrometry and redox proteomics: Applications in disease. Mass Spectrom Rev 2014;33:277-301.

11 Abbott A: And now for the proteome. Nature 2001;409:747.

$12 \mathrm{Xu} \mathrm{S}$, Li Z, Zhai C, Fan W: Proteomics analysis of rat chondrocytes under periodic mechanical stress. Jiangsu Med J 2016;42:869-871.

13 Gingras AC, Wong CJ: Proteomics approaches to decipher new signaling pathways. Curr Opin Struct Biol 2016;41:128-134.

14 Karabulut NP, Frishman D: Sequence- and Structure-Based analysis of Tissue-Specific phosphorylation sites. PLoS One 2016;11:e157896.

15 Karp NA, Spencer M, Lindsay H, O’Dell K, Lilley KS: Impact of replicate types on proteomic expression analysis. J Proteome Res 2005;5:1867-1871.

16 Müller C, Khabut A, Dudhia J, Reinholt FP, Aspberg A, Heinegård D, Önnerfjord P: Quantitative proteomics at different depths in human articular cartilage reveals unique patterns of protein distribution. Matrix Biol 2014;40:34-45.

-17 Wisniewski JR, Zougman A, Nagaraj N, Mann M: Universal sample preparation method for proteome analysis. Nat Methods 2009;6:359-362.

-18 Larsen MR, Thingholm TE, Jensen ON, Roepstorff P, Jorgensen TJ: Highly selective enrichment of phosphorylated peptides from peptide mixtures using titanium dioxide microcolumns. Mol Cell Proteomics 2005;4:873-886.

19 Pu X, Oxford JT: Proteomic analysis of engineered cartilage. Methods Mol Biol 2015;1340:263-278.

-20 Xie SW, Li GT, Qu LJ, Cao Y, Wang Q, Zhou JY, Zhong RH, Guo XJ , Zhu Y: Identification of new epididymal luminal fluid proteins involved in sperm maturation in infertile rats treated by dutasteride using iTRAQ. Molecules 2016;21:5.

21 Unwin RD, Griffiths JR, Whetton AD: Simultaneous analysis of relative protein expression levels across multiple samples using iTRAQ isobaric tags with 2D nano LC-MS/MS. Nat Protoc 2010;5:1574-1582.

22 Hu X, Li N, Wu L, Li C, Li C, Zhang L, Liu T, Wang W: Quantitative iTRAQ-based proteomic analysis of phosphoproteins and ABA-regulated phosphoproteins in maize leaves under osmotic stress. Sci Rep 2015;5:15626.

23 Su Z, Zhu H, Zhang M, Wang L, He H, Jiang S, Hou FF, Li A: Salt-induced changes in cardiac phosphoproteome in a rat model of chronic renal failure. PLoS One 2014;9:e100331.

24 Gan CS, Chong PK, Pham TK, Wright PC: Technical, experimental, and biological variations in isobaric tags for relative and absolute quantitation (iTRAQ). J Proteome Res 2007;6:821-827.

25 Moulder R, Lonnberg T, Elo LL, Filen JJ, Rainio E, Corthals G, Oresic M, Nyman TA, Aittokallio T, Lahesmaa R: Quantitative proteomics analysis of the nuclear fraction of human CD4+ cells in the early phases of IL-4induced Th2 differentiation. Mol Cell Proteomics 2010;9:1937-1953. 


\section{Cellular Physiology Cell Physiol Biochem 2017;44:1578-1590 \begin{tabular}{l|l|l}
\hline and Biochemistry 10.1159/000485652 & $\begin{array}{l}\text { C } 2017 \text { The Author(s). Published by S. Karger AG, Basel } \\
\text { www.karger.com/cpb }\end{array}$ \\
\hline
\end{tabular}

-26 Livak KJ, Schmittgen TD: Analysis of relative gene expression data using real-time quantitative PCR and the 2- $\Delta \Delta$ Ct Method. Methods 2001;25:402-408.

27 MacDonald BT, Tamai K, He X: Wnt/beta-catenin signaling: Components, mechanisms, and diseases. Dev Cell 2009;17:9-26.

28 Guo Y, Xiao L, Sun L, Liu F: Wnt/beta-catenin signaling: A promising new target for fibrosis diseases. Physiol Res 2012;61:337-346.

29 Liu W, Hao J, Zhu L, Li F, Liu Q, Liu S, Zhao S, Li H, Duan H: Phospho-GSK-3beta is involved in the highglucose-mediated lipid deposition in renal tubular cells in diabetes. Int J Biochem Cell Biol 2013;45:20662075.

-30 Grassilli E, Ianzano L, Bonomo S, Missaglia C, Cerrito MG, Giovannoni R, Masiero L, Lavitrano M: GSK3A is redundant with GSK3B in modulating drug resistance and chemotherapy-induced necroptosis. PLoS One 2014;9:e100947.

-31 Wolf A, Ackermann B, Steinmeyer J: Collagen synthesis of articular cartilage explants in response to frequency of cyclic mechanical loading. Cell Tissue Res 2007;327:155-166.

32 Mak KM, Png CY, Lee DJ: Type v collagen in health, disease, and fibrosis. Anat Rec 2016;299:613-629.

33 Piersma B, Bank RA, Boersema M: Signaling in fibrosis: TGF-beta, WNT, and YAP/TAZ converge. Front Med 2015;2:59.

34 Lam AP, Gottardi CJ: Beta-catenin signaling: A novel mediator of fibrosis and potential therapeutic target. Curr Opin Rheumatol 2011;23:562-567.

-35 Singh SP, Tao S, Fields TA, Webb S, Harris RC, Rao R: Glycogen synthase kinase-3 inhibition attenuates fibroblast activation and development of fibrosis following renal ischemia-reperfusion in mice. Dis Model Mech 2015;8:931-940.

-36 Yuan X, Liu H, Huang H, Liu H, Li L, Yang J, Shi W, Liu W, Wu L: The key role of canonical wnt/beta-catenin signaling in cartilage chondrocytes. Curr Drug Targets 2016;17:475-484.

37 Http://www.uniprot.org/uniprot/P18266.

-38 McCubrey JA, Steelman LS, Bertrand FE, Davis NM, Abrams SL, Montalto G, D’Assoro AB, Libra M, Nicoletti F, Maestro R, Basecke J, Cocco L, Cervello M, Martelli AM: Multifaceted roles of GSK-3 and Wnt/beta-catenin in hematopoiesis and leukemogenesis: Opportunities for therapeutic intervention. Leukemia 2014;28:15-33.

-39 Darling EM, Athanasiou KA: Rapid phenotypic changes in passaged articular chondrocyte subpopulations. J Orthop Res 2005;23:425-432.

40 Schnabel M, Marlovits S, Eckhoff G, Fichtel I, Gotzen L, Vecsei V, Schlegel J: Dedifferentiation-associated changes in morphology and gene expression in primary human articular chondrocytes in cell culture. Osteoarthr Cartilage 2002;10:62-70.

41 Liang W, Zhu C, Liu F, Cui W, Wang Q, Chen Z, Zhou Q, Xu S, Zhai C, Fan W: Integrin beta1 gene therapy enhances in vitro creation of Tissue-Engineered cartilage under periodic mechanical stress. Cell Physiol Biochem 2015;37:1301-1314.

42 Behery O, Siston RA, Harris JD, Flanigan DC: Treatment of cartilage defects of the knee. Clin J Sport Med 2014;24:21-30.

43 Smith GD, Knutsen G, Richardson JB: A clinical review of cartilage repair techniques. J Bone Joint Surg BR 2005;87:445-449.

-44 Oussedik S, Tsitskaris K, Parker D: Treatment of articular cartilage lesions of the knee by microfracture or autologous chondrocyte implantation: A systematic review. Arthroscopy 2015;31:732-744.

45 Li Z, Zhu T, Fan W: Osteochondral autograft transplantation or autologous chondrocyte implantation for large cartilage defects of the knee: A meta-analysis. Cell Tissue Bank 2016;17:59-67.

46 Bentley G, Biant LC, Vijayan S, Macmull S, Skinner JA, Carrington RWJ: Minimum ten-year results of a prospective randomised study of autologous chondrocyte implantation versus mosaicplasty for symptomatic articular cartilage lesions of the knee. J Bone Joint Surg AM 2012;94-B:504-509.

47 Benya PD, Padilla SR, Nimni ME: Independent regulation of collagen types by chondrocytes during the loss of differentiated function in culture. Cell 1978;15:1313-1321. 